\title{
Coercive field and energy barriers in partially disordered FePt nanoparticles
}

Cite as: J. Appl. Phys. 105, 07B514 (2009); https://doi.org/10.1063/1.3067570

Submitted: 16 September 2008 . Accepted: 02 November 2008 . Published Online: 09 February 2009

G. R. Aranda, O. Chubykalo-Fesenko, R. Yanes, J. González, J. J. del Val, R. W. Chantrell, Y. K. Takahashi, and K. Hono

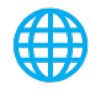

\section{ARTICLES YOU MAY BE INTERESTED IN}

Switching and thermal stability properties of bilayer thin films: Single versus multigrain cases Journal of Applied Physics 103, 07F505 (2008); https://doi.org/10.1063/1.2829584

Micromagnetic modeling of laser-induced magnetization dynamics using the Landau-LifshitzBloch equation

Applied Physics Letters 91, 232507 (2007); https://doi.org/10.1063/1.2822807

Multiscale versus micromagnetic calculations of the switching field reduction in FePt / FeRh bilayers with perpendicular exchange spring

Journal of Applied Physics 97, $10 J 101$ (2005); https://doi.org/10.1063/1.1844931

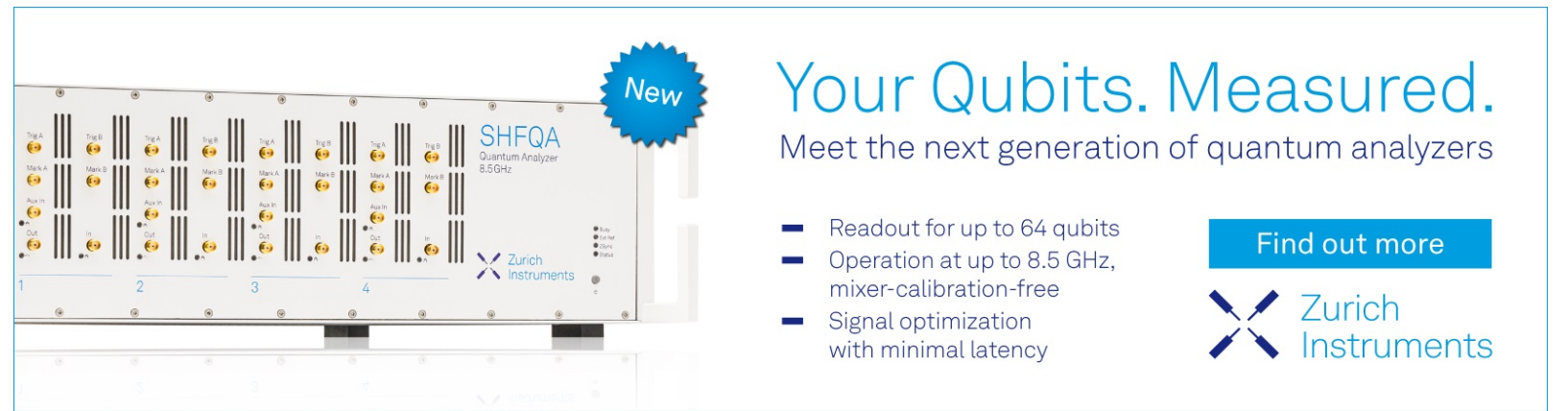




\title{
Coercive field and energy barriers in partially disordered FePt nanoparticles
}

\author{
G. R. Aranda, ${ }^{1, a)}$ O. Chubykalo-Fesenko, ${ }^{2}$ R. Yanes, ${ }^{2}$ J. González, ${ }^{3}$ J. J. del Val, ${ }^{1,3}$ \\ R. W. Chantrell, ${ }^{4}$ Y. K. Takahashi, ${ }^{5}$ and K. Hono ${ }^{5}$ \\ ${ }^{1}$ Unidad de Física de Materiales, Centro Mixto CSIC-UPV/EHU, San Sebastián, Gipuzkoa 20018, Spain \\ ${ }^{2}$ Instituto de Ciencia de Materiales de Madrid, CSIC, Cantoblanco, Madrid 28049, Spain \\ ${ }^{3}$ Dpto. de Física de Materiales, Facultad de Química, UPV/EHU, San Sebastián, Gipuzkoa 20018, Spain \\ ${ }^{4}$ Department of Physics, University of York, York YO10 5DD, United Kingdom \\ ${ }^{5}$ National Institute for Materials Science, Tsukuba 305-0047, Japan
}

(Presented 13 November 2008; received 16 September 2008; accepted 2 November 2008; published online 9 February 2009)

\begin{abstract}
Partially disordered FePt nanoparticles prepared by the sputtering method and protected by $\mathrm{Al}_{2} \mathrm{O}_{3}$ constitute an interesting realization of graded media, recently suggested for future generations of magnetic recording. By depositing the alumina layer, the particle is partially disordered with gradually varying properties. The current work comprises a comparison between experimental data and atomistic modeling results of the coercive field and energy barriers in FePt particles with gradually spatially varying properties, specifically the magnetization, anisotropy, and exchange constants. From our modeling we conclude that the magnetization reversal processes for dynamic reversal at the coercive field and for the zero field energy barrier involve different reversal modes. The coercive field decreases as a function of disordering length up to $2 \mathrm{~T}$ and is in good agreement with measured values. The zero field energy barrier, except for highly disordered particles, is almost independent of the disordering length, retaining the high values required for thermal stability. (C) 2009 American Institute of Physics. [DOI: 10.1063/1.3067570]
\end{abstract}

FePt is an important material for future magnetic recording media due to its large magnetocrystalline anisotropy in the $L l_{0}$ phase. Its maximum anisotropy value could be as large as $10^{8} \mathrm{erg} / \mathrm{cm}^{3}$ and this would provide the required thermal stability even for the small grain sizes required for ultrahigh density recording. However, the coercive field necessary to reverse the magnetization in FePt nanograins can be as high as $20 \mathrm{~T}$, which is not suitable for conventional magnetic recording heads. This problem can be substantially overcome by adding a magnetically soft layer whose role is to decrease the switching field. ${ }^{1,2}$ However, it should be noted that for all media designs the energy barrier in zero field must remain high in order to ensure thermal stability of the written information. Suess ${ }^{3}$ suggested the use of a multilayer system (graded media) and showed that in this case the coercive field could be decreased further without significant deterioration of the energy barrier in zero field. The simulation of the recording process has confirmed the performance of such media for new generations of magnetic recording. ${ }^{4}$

An interesting and novel realization of the graded media concept consists of partially disordered FePt nanoparticles, prepared by sputtering, with a protective layer of $\mathrm{Al}_{2} \mathrm{O}_{3} \cdot{ }^{5,6}$ The nanoparticles have an average size of $10 \mathrm{~nm}$ with a small dispersion of $2.8 \mathrm{~nm}$ and are well separated from one another so that the interactions are negligible. During deposition of the alumina layer, the $\mathrm{Al}_{2} \mathrm{O}_{3}$ partially penetrates the hard FePt phase to an extent which decreases with distance into the FePt layer. ${ }^{6}$ This results in a low anisotropy phase with a graded anisotropy arising from the decrease in pen-

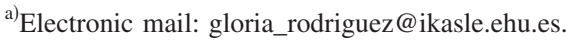

etration of $\mathrm{Al}_{2} \mathrm{O}_{3}$ with depth. By this technique it is possible to prepare particles with the same height but different thicknesses of the graded anisotropy layer. It was shown that the coercive field decreases as a function of the graded layer thickness. The energy barriers were extracted from the dynamic coercivity measurements using the Sharrock law. ${ }^{7}$ The present work consists of modeling of the experimental results ${ }^{5}$ of coercive field and energy barriers in partially disordered FePt particles.

In this paper we present an atomistic model of the magnetic properties of graded media. In our model we consider for simplicity cylindrical nanoparticles with diameter $D$ $=10 \mathrm{~nm}$ corresponding to the experimental results. ${ }^{5,6}$ The total particle height was kept constant at $H=5.32 \mathrm{~nm}$. Note that unlike the original proposal for the graded media, ${ }^{3}$ where long nanograins (with the height of about $20 \mathrm{~nm}$ ) were considered, these nanoparticles have the opposite aspect ratio: the in-plane $(X Y)$ dimensions are larger than the out-of plane $(Z)$ one.

The FePt easy axis is directed perpendicular to the plane, i.e., parallel to the cylinder axis (Z-direction). The particle was discretized into atomistic cubic discretization cells with lattice parameter $a=0.38 \mathrm{~nm}$. The magnetic moments are placed at the Fe sites only in the spirit of Ref. 8. The hard phase magnetization and anisotropy parameters were taken to be $M_{s}^{\text {hard }}=1100 \mathrm{emu} / \mathrm{cm}^{3}, K^{\text {hard }}=4 \times 10^{7} \mathrm{erg} / \mathrm{cm}^{3}$. As for the exchange parameter, we have considered two models with $J_{\mathrm{ex}}^{\text {hard }}=3.1 \times 10^{-14} \mathrm{erg}$ and $7.7 \times 10^{-14} \mathrm{erg}$. The former corresponds to the average value estimated from the Curie temperature and the latter corresponds to the FePt in-plane exchange estimated from first principles calculations. ${ }^{8}$ These values are used as lower and upper limits for the in-plane 


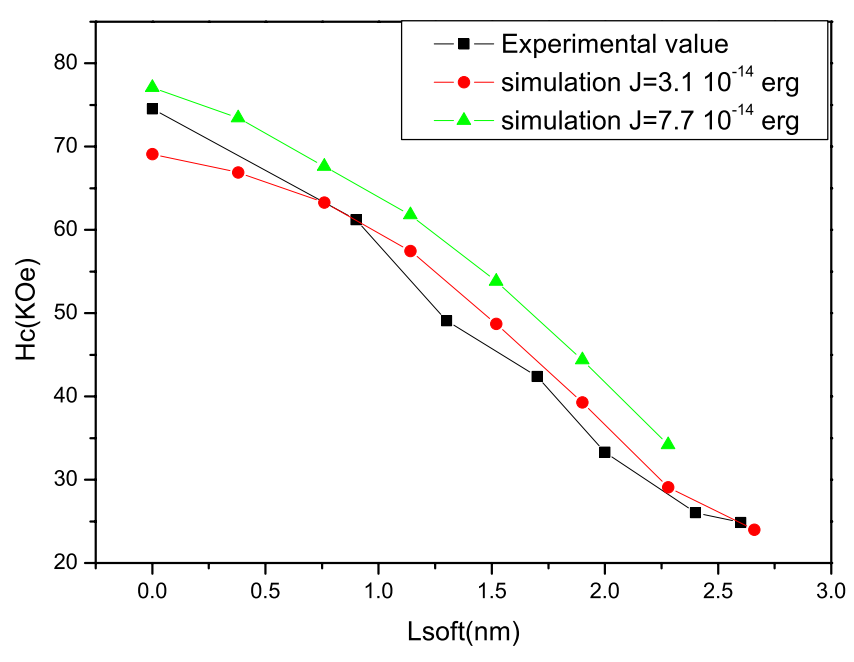

FIG. 1. (Color online) Comparison between measured and modeled coercive field values as a function of the disordering length in FePt nanoparticle with $J_{\mathrm{ex}}=3.1 \times 10^{-14} \mathrm{erg}, \quad L_{\mathrm{soft}}^{0}=2.66 \mathrm{~nm}$ and $J_{\mathrm{ex}}=7.7 \times 10^{-14} \mathrm{erg}, \quad L_{\text {soft }}^{0}$ $=2.28 \mathrm{~nm}$.

exchange values. In the first case the domain wall having the width of $4.49 \mathrm{~nm}$ can fit into the nanoparticle in the direction parallel to the cylinder axis, which is not true for the second case, where the domain wall width is $7.07 \mathrm{~nm}$.

We considered nanoparticles of thickness $H=L_{\text {soft }}$ $+L_{\text {hard }}$ with linearly varying properties such as magnetization, anisotropy within the graded layer of thickness $L_{\text {soft }}$, specifically in the region $L_{\text {hard }}<z<H: \quad K^{\text {soft }}(z)=K^{\text {hard }}$ $-\left[\left(K^{\text {hard }}-K_{0}^{\text {soft }}\right) / L_{\text {soft }}^{0}\right]\left(z-L_{\text {hard }}\right)[$ and similar dependence for $\left.J^{\text {soft }}(z)\right]$. Inside the region $z<L_{\text {hard }}$ the parameters were kept those of the hard phase. The fully disordered properties were taken as $K_{\text {soft }}^{0}=4 \times 10^{5} \mathrm{erg} / \mathrm{cm}^{3}$ and $J_{\text {soft }}^{0}=J_{\text {hard }}^{0} / 10$. The gradual change in the magnetization was found to have little influence on the magnetic properties. We use an atomistic model with the Heisenberg form for exchange, and the magnetostatic fields were calculated via the fast Fourier transform algorithm. The hysteresis cycle was calculated by integration of the Landau-Lifshitz-Gilbert equation. For the energy barrier calculation we used the Lagrangian multiplier technique ${ }^{9}$ constraining the average angle of the magnetization to a particular direction $\theta_{0}$; variation in $\theta_{0}$ maps out the energy landscape from which the energy barrier in zero field is calculated.

First we present calculations of the coercive field with a field applied parallel to the easy axis. Calculations were made as a function of the length $L_{\text {soft }}$ of the graded region. In Fig. 1 it can be seen that with increasing disordered layer thickness, the coercive field decreases to $H_{c} \approx 2 \mathrm{~T}$ for nanoparticles with half of their height gradually disordered. We also note the remarkable agreement between simulation and experimental values, the best agreement corresponding to the parameters $L_{\text {soft }}^{0}=2.28 \mathrm{~nm}$ for high $J$ value and $L_{\text {soft }}^{0}$ $=2.66 \mathrm{~nm}$ for low $J$ value. In what follows, we will use these values of the parameters. All the hysteresis loops have a square shape. This suggests (quasi)coherent magnetization reversal in all cases. However, dynamically the processes are slightly different. The process is much more collective for larger exchange value, being completely coherent if no soft

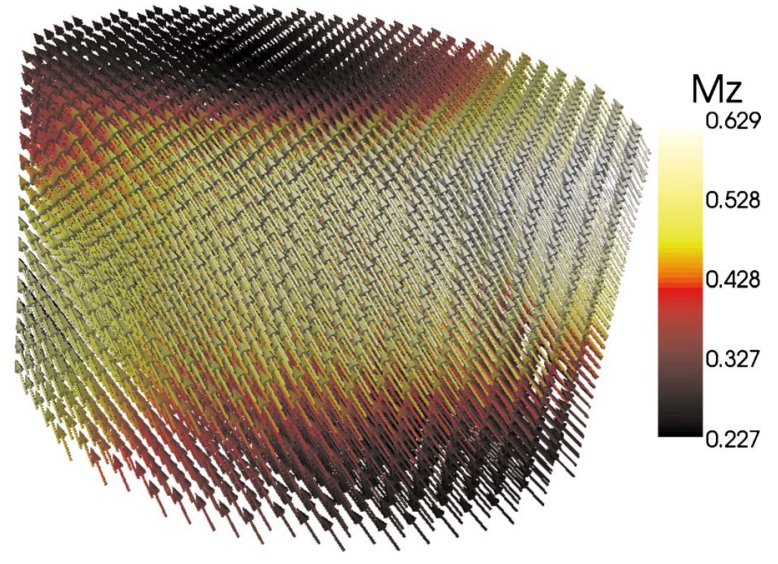

FIG. 2. (Color online) Dynamical magnetization configuration during the hysteresis process for a magnetically hard FePt nanoparticle with $J_{\text {ex }}=3.1$ $\times 10^{-14}$ erg showing the magnetization nucleation on both top and upper layers. The gray map represents the $M_{z}$ magnetization value.

layer is present. In a completely hard particle, for lower exchange value the demagnetization process starts simultaneously on both top and bottom layers (see Fig. 2) and propagates to the particle center. However, the addition of even one soft layer makes the top and bottom parts nonequivalent. In this case the demagnetization dynamically always starts on the top (soft) layer (see Fig. 3). In the low exchange case during this process a $180^{\circ}$ wall is created. Unlike the high exchange value, in the case of the low exchange, the demagnetization process is nonhomogeneous in plane.

Next, we investigate the zero field energy barriers of the disordered FePt nanoparticles. Figure 4 presents the comparison between experimental and simulated values of the energy barriers as a function of the height of the disordered region. Note that these values are only slightly dependent on the disorder and well preserve the thermal stability of the particles for large time scale.

Figure 5 represents the zero-field thermally activated reversal mode (saddle point or energy barrier magnetization configuration). In the originally suggested graded media the thermal mode configuration is a domain wall in the hard

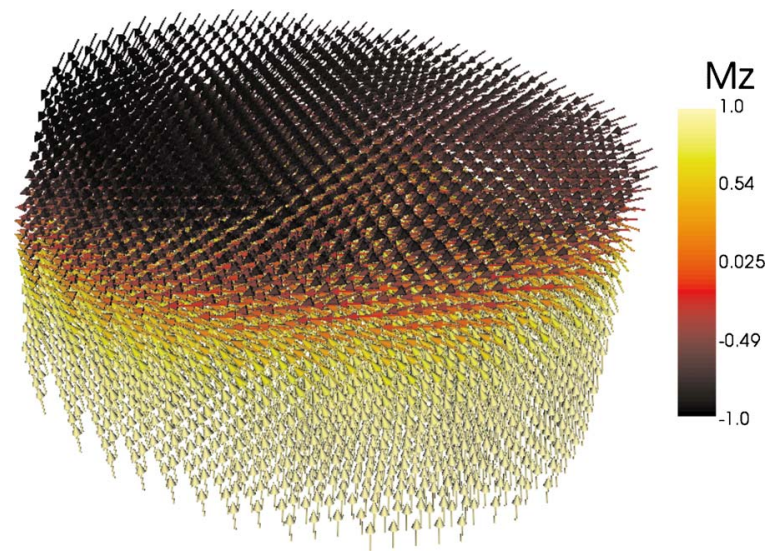

FIG. 3. (Color online) Dynamical magnetization configuration during the hysteresis process in a disordered FePt nanoparticle with $J_{\mathrm{ex}}=3.1$ $\times 10^{-14}$ erg and $L_{\text {soft }}=2.28 \mathrm{~nm}$. The gray map represents the $M_{z}$ magnetization value. 


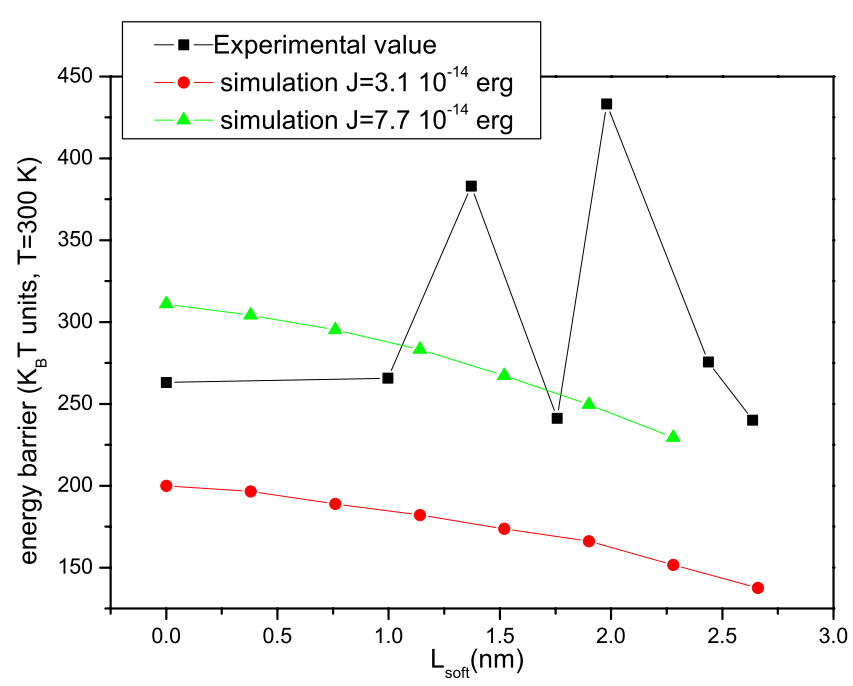

FIG. 4. (Color online) Comparison between calculated and experimental energy barrier values (in $k_{B} T$ units, $T=300^{\circ} \mathrm{K}$ ) as a function of the disordering length in FePt nanoparticle with $J_{\mathrm{ex}}=3.1 \times 10^{-14}$ erg and $J_{\mathrm{ex}}=7.7$ $\times 10^{-14}$ erg.

material in the direction of the easy axis (perpendicular to the film), ${ }^{3}$ having homogeneous magnetization in the in $X Y$ direction. In the present case the domain wall cannot be fitted perpendicularly in the particle for large exchange value but in principle, this is possible for a smaller exchange. However, in both cases for zero-field thermal reversal the particle prefers to be divided into an in-plane $(X Y)$ domain configuration. For smaller exchange value the energy of such configuration appears to be smaller than that of the perpendicu-

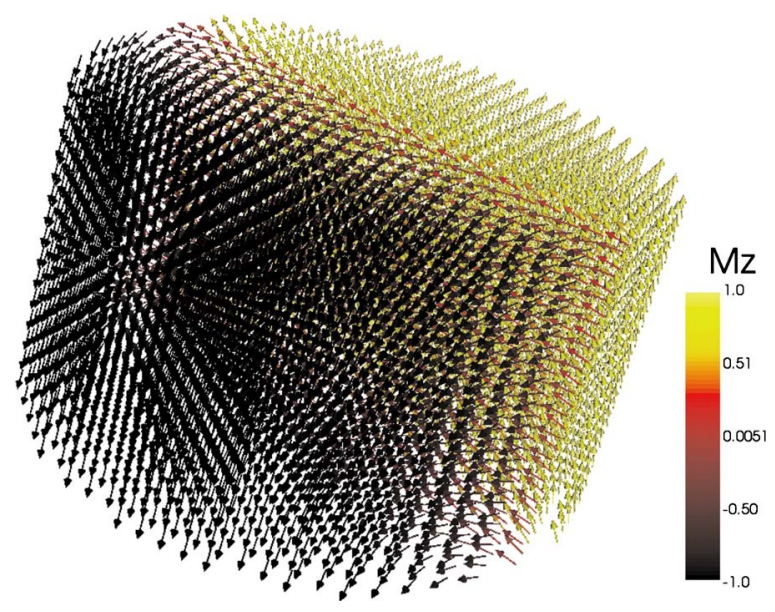

FIG. 5. (Color online) Magnetization configuration corresponding to the thermal reversal mode in a completely hard FePt nanoparticle with $J_{\text {ex }}$ $=3.1 \times 10^{-14} \mathrm{erg}$. The gray map represents the $M_{z}$ magnetization value. lar domain wall due to the nanoparticle dimensions. The character of the saddle point with a wall dividing the particle into two vertical domains is shown in Fig. 5. We note that the best fit in Fig. 4 is obtained for the larger value of exchange coupling, in contrast to the data of Fig. 1. This apparent discrepancy may arise from the fact that experimentally the energy barriers were evaluated using the Sharrock law, ${ }^{7}$ which is probably not applicable for the present case due to the highly inhomogeneous character of the saddle point.

In conclusion, partially disordered FePt nanoparticles represent an interesting realization of graded media. Partial disordering by the penetration of $\mathrm{Al}_{2} \mathrm{O}_{3}$ into the hard $\mathrm{FePt}$ layer provides a decrease in the coercive field to a value of 2 $\mathrm{T}$, essentially without significant decrease in the energy barrier. The oblate spheroidal geometry of nanoparticles is different to that of the classical magnetic recording grains. The consequence of this is a different saddle point configuration during thermally activated reversal in zero field, where the particle is divided in two vertical domains with opposite magnetization direction. The associated energy barrier could be even larger than that of the graded media suggested in Ref. 3. The field-induced reversal mode has a different nature and, when the soft phase is increased, ends being a $180^{\circ}$ domain wall propagating in the $Z$-direction. The idea of partial disordering by protecting layer, the design, and the conclusions of the present paper could be directly applicable to the bit-patterned media based on FePt.

G.R.A. wants to thank her postdoctoral grants conceded by the Basque Government and the CSIC (JAE fellowship). The SGI/IZO-SGIker UPV/EHU is gratefully acknowledged for allocation of computational resources. This work has also been supported by the Spanish Ministry of Science and Education (Project No. NAN-2004-09125-C07-06) and by the project NANOMAGNET from Comunidad de Madrid.

${ }^{1}$ J.-U. Thiele, S. Maat, and E. E. Fullerton, Appl. Phys. Lett. 82, 2859 (2003).

${ }^{2}$ K. Yu. Guslienko, O. Chubykalo-Fesenko, O. Mryasov, R. Chantrell, and D. Weller, Phys. Rev. B 70, 104405 (2004).

${ }^{3}$ D. Suess, Appl. Phys. Lett. 89, 113105 (2006).

${ }^{4}$ A. Goncharov, T. Schrefl, G. Hrkac, J. Dean, S. Bance, D. Suess, O. Ertl, F. Dorfbauer, and J. Fidler, Appl. Phys. Lett. 91, 222502 (2007).

${ }^{5}$ Y. K. Takahashi, K. Hono, S. Okamoto, and O. Kitakami, J. Appl. Phys. 100, 074305 (2006).

${ }^{6}$ Y. K. Takahashi and K. Hono, Appl. Phys. Lett. 84, 383 (2004).

${ }^{7}$ M. P. Sharrock, J. Appl. Phys. 76, 6413 (1994).

${ }^{8}$ O. N. Mryasov, U. Nowak, K. Y. Guslienko, and R. W. Chantrell, Europhys. Lett. 69, 805 (2005).

${ }^{9}$ E. Paz, F. Garcia-Sanchez, and O. Chubykalo-Fesenko, Physica B 403, 330 (2008). 